\title{
Pre-seismic variations of atmospheric radon activity as a possible reason for abnormal atmospheric effects
}

\author{
Vadim V. Surkov
}

National Research Nuclear University MEPhI, Moscow, Russia

\author{
Article history \\ Received June 15, 2015; accepted October 2, 2015. \\ Subject classification: \\ Atmosphere, Aerosols, Radioactivity and isotopes, Earthquake source and dynamics, Heat generation and transport.
}

\begin{abstract}
Possible atmospheric effects associated with pre-seismic variations of atmospheric radon concentration occasionally observed prior to earthquake occurrence are subjected to theoretical study in order to assess their potential effectiveness. Altitude distribution of atmospheric conductivity is examined as a function of radon and aerosol concentrations. Horizontal components of atmospheric electric fields and currents, which can arise near a boundary of seismo-active region, are estimated. We speculated that changes in radon-induced air ionization can have an impact on fairweather spectra of ULF electric pulsations. Particular emphasis has been placed on hypothesis for correlation between variations of pre-seismic radon activity and upward thermal radiation measured by satellite over the high seismicity regions. This hypothesis is based on assumption that the radon-induced ionization of atmospheric surface layer can trigger water vapor condensation which in turn results in release of the vaporization heat. Our analysis has shown that the upward thermal flux has to be many orders of magnitude lower than that predicted on the basis of this hypothesis.
\end{abstract}

\section{Introduction}

It is generally believed that the main ionization of the atmospheric surface layer is due to galactic cosmic rays and radiation as well as the naturally occurring radioactive elements naturally found in the air. Shortwave solar radiation and energetic particles of the solar wind predominately affect the upper atmosphere above altitudes of $50 \mathrm{~km}$. A mean rate of ion production under the cosmic rays is approximately the same in all points of the Earth surface at all times. At sea level its value is $\gamma_{c}=1.5-1.7$ ion pairs per $\mathrm{cm}^{3}$ per second [e.g., Tverskoy 1962, Chalmers 1967, Israël 1970]. The radioactive elements contained in the surface layer of the soil play insignificant role. For the most the air ionization is due to radioactive atoms which first enter to the atmosphere from the soil and then accumulate in the surface layer. On land, the ionization rate due to radioactive elements that are naturally present in the atmospheric surface layer is estimated to be $\gamma_{r}=8-10$ ion pairs $/\left(\mathrm{cm}^{3} \mathrm{~s}\right)$ although this value is slightly lower near the ocean surface. According to other estimates both the mechanisms, i.e. the cosmic rays and radioactivity, make approximately equal contribution to the ion production process in the surface layer [e.g., Tverskoy 1962, Zhang et al. 2011].

The ionization rate $\gamma_{c}$ due the cosmic rays increases with increasing altitude and it reaches a peak value at the altitudes 14-15 km [e.g., Tverskoy 1962]. Alternatively, the number density of the radioactive atoms decreases with increasing altitude thereby producing the decrease in the ionization rate $\gamma_{r}$ in such a way that $\gamma_{r}$ becomes practically negligible at the altitudes $4-5 \mathrm{~km}$.

A significant factor, which may greatly affect the background radiation, is the heavy gas radon that has the tendency to penetrate permanently along the systems of capillaries and cracks through the rocks into the soil and air. Among many radioactive rocks are uranium-containing rock, granite, gneiss, shale, tuff, metalbearing ore, phosphorite, and potassium-containing rock. The ${ }_{86}^{222} \mathrm{Rn}$ nuclei are efficient sources of the air ionization since their half-life is about 3.82 days [e.g., Protection against Radon-222 at Home and at Work, 1994]. The half-life of other radon isotopes; that is, ${ }_{86}^{219} \mathrm{Rn}$ and ${ }_{86}^{222} \mathrm{Rn}$ is much smaller than the above value. The radon concentration in the air depends on a host of factors such as local species of rocks, the configuration and state of soil surface, the possibility for removal of radioactive ingredients due to rainout, radon flux from depth, the rate of radon dissipation in the atmosphere which in turn depends on the meteorological conditions including the direction and strength of wind, pressure, air humidity and etc.

The enhancement of background radiation has been occasionally observed in the regions of tectonic faults of the Earth crust [Toutain and Baubron 1998]. Some researchers have speculated that the abnormal 
variations of radon concentration in the atmospheric surface layer can be due to the earthquake preparation processes [Pierce 1976]. A review of anomalous radon precursors have been reported for a very wide range of earthquake magnitudes [Cicerone et al. 2009]. For example, Virk and Singh [1994] have reported that radon concentration in the soil gases and in the spring water suddenly increased by 2.5 and 1.5 times a week before Uttarkashi earthquake. These measurements have been pursued at the epicentral distance of $300 \mathrm{~km}$. Yasuoka et al. [2009] have detected a gradual increase in the atmospheric radon emanation two months before Kobe earthquake occurred on January 17, $1995\left(M_{w}=6.9\right)$. The radon volumetric activity was increased up to 20 $\mathrm{Bq} / \mathrm{m}^{3}$ just before an earthquake and it was approximately doubled as compared to the background level. It should be noted that the evidence of pre-seismic radon anomalies in the atmosphere are fewer than those detected directly in the soil or in indoor locations [e.g., Cicerone et al. 2009]. However, some researchers have not found any statistically significant changes in radon concentration before earthquakes [Geller 1997, Inan et al. 2008]. Whether the radon concentration variation is a plausible precursor of impending earthquake or not is still an open question. Also the answer to this question is likely to be dependent from the properties of seismogenetic fault.

\section{Local changes of electrical resistance of the at- mosphere}

The electric conductivity of the air increases approximately exponentially with height if standard conditions prevail. In this notation the surface atmospheric layer makes a major contribution to the total electric resistance of the atmosphere. The conductivity of the lower atmosphere is predominately due to small/light ions that have the greatest mobility. The small ions build up as a result of the attachment of a few neutral molecules to the primary ions, which in turn are caused by the cosmic rays and ionizing radiation of the radioactive substances. The small ions many times collide with other particles and take part into enormous number of chemical reactions during their life-time. The composition and number of molecules entering into the composition varies with time. The mean number of these molecules depends on air humidity. On the average, 10-20 neutral molecules are held around each positive ion while a negative small ion contains 5-10 neutral molecules [e.g., Tverskoy 1962, Chalmers 1967, Israël 1970].

To estimate the radon variations impact on the electric resistance of the surface atmospheric layer, we first consider the simplified model of the atmosphere in which there are only two species of opposite-charged small ions. Suppose that the small ions may disappear either by direct recombination with oppositely charged small ions or by attachment to aerosol particles. Taking into account that the concentration $n_{i}$ of both ion species are approximately the same, we come to the following equation [e.g., Israël 1970]

$$
\frac{\partial n_{i}}{\partial t}=\nabla \cdot \mathbf{J}+\gamma_{c}+\gamma_{r}-\alpha n_{i}^{2}-\beta n_{i} n_{a}
$$

where $\alpha$ denotes the ion-ion volumetric recombination coefficient, $n_{a}$ is aerosol concentration, $\beta$ is the ionaerosol attachment coefficient, and $\mathbf{J}$ stands for the small ion flux density due to entrainment of small ions with air fluxes, small ion diffusion, small ion drift in electric field and etc. Here we shall examine the quasi-steady small ion distribution in which $\partial_{t} n_{i}=0$ and $\mathbf{J}$ is assumed to be constant value. It follows from Equation (1) then [e.g., Harrison et al. 2010]

$$
n_{i}=\frac{1}{2 \alpha}\left\{\left(\beta^{2} n_{a}^{2}+4 \alpha\left[\gamma_{c}+\gamma_{r}\right]\right)^{1 / 2}-\beta n_{a}\right\}
$$

The values of parameters entering Equation (2) can strongly vary with altitude. Whenever the atmosphere is in thermodynamic equilibrium, the concentrations of the aerosol particles and radon atoms as well as the rate $\gamma_{r}$ of ion production decrease with altitude according to Boltzmann distribution. By contrast, the rate $\gamma_{c}$ ion production by cosmic rays increases approximately exponentially with altitude $z$ [Wåhlin 1994]. Actually, all these altitude dependences can be approximated by exponential functions as follows

$$
\begin{aligned}
& \gamma_{c}=\gamma_{c 0} \exp \left(z / l_{c}\right), \\
& \gamma_{r}=\gamma_{r 0} \exp \left(-z / l_{r}\right), \\
& n_{a}=n_{a 0} \exp \left(-z / l_{a}\right)
\end{aligned}
$$

where $\gamma_{c 0}$ and $\gamma_{r 0}$ are the ionization rate at the sea level. Here we made use of the following abbreviations $l_{r}=$ $k_{B} T /\left(m_{r} g\right)$ and $l_{a}=k_{B} T /\left(m_{a} g\right)$, where $k_{B}$ is Boltzmann constant, $g$ is acceleration of free fall, $m_{r}$ is a mass of radon atom, $m_{a}$ is an average mass of aerosol particles, and $l_{c}$ is an unknown parameter that will be estimated later. Substituting the temperature $T=293 \mathrm{~K}$ into Equation (3), yields $l_{r} \approx 1.1 \mathrm{~km}$. Taking into account that the typical sizes of the aerosols possess value within 0.002-1 $\mu \mathrm{m}$ [e.g., Ivlev and Dovgalyuk 1999, Kondratyev et al. 2006], the length $l_{a}$ is no greater than several meters even for the smallest aerosols. These values can 
serve as rough estimates since the actual altitude distributions of the radon atoms, small ions and aerosols can be subjected to local meteorological conditions, air flow, turbulent and convective gas motion, which can drag these particles, and many other factors. Considering singly charged small ions, the air conductivity is given by $\sigma=2 \mu_{i} n_{i} e$, where $e$ denotes the elementary charge. The small ion mobility $\mu_{i}$ is inversely proportional to the concentration of neutral molecules which in turn exponentially decreases with altitude. Whence it follows that $\mu_{i}=\mu_{i 0} \exp (z / H)$, where $\mu_{i 0}$ is the small ion mobility at the sea level and $H \approx 8 \mathrm{~km}$ denotes the scale-height of an isothermal atmosphere. Combining Equations (2) and (3) with the above equations, we obtain the air conductivity

$$
\begin{gathered}
\sigma=\frac{e \mu_{i 0} n_{a 0} \beta}{\alpha} \exp \left(\frac{z}{H}-\frac{z}{l_{a}}\right) \int\left\{\left(1+\frac{4 \alpha}{n_{a 0}^{2} \beta^{2}} \exp \left[\frac{2 z}{l_{a}}\right] \times\right.\right. \\
\left.\left.\left[\gamma_{c 0} \exp \left(\frac{z}{l_{c}}\right)+\gamma_{r 0} \exp \left(-\frac{z}{l_{r}}\right)\right]\right)^{1 / 2}-1\right\} .
\end{gathered}
$$

To make an estimate, we use the following numerical values [e.g., Pierce 1976, Harrison et al. 2010]: $\mu_{0} \approx 1.2 \cdot 10^{-4} \mathrm{~m}^{2} \mathrm{~V}^{-1} \mathrm{~s}^{-1}, \alpha=1.6 \times 10^{-12} \mathrm{~m}^{3} \mathrm{~s}^{-1}, \beta=4 \times 10^{-11}$ $\mathrm{m}^{3} \mathrm{~s}^{-1}$ (for the aerosol size of $0.2-0.25 \mu \mathrm{m}$ ). At the ground level the aerosol concentration $n_{a 0}$ varies from $200 \mathrm{~cm}^{-3}$ for clean air to $1.5 \times 10^{3} \mathrm{~cm}^{-3}$ for dusty air [e.g., Harrison et al. 2010]. In such a case the condition $\beta^{2} n_{a}^{2} \gg$ $4 \alpha\left(\gamma_{c}+\gamma_{r}\right)$ is valid for the atmospheric surface layer. In fact this means that one may neglect the term $\alpha n_{i}^{2}$ which describes the small ion recombination in Equation (1). In this extreme case Equation (4) for air conductivity is reduced to

$$
\sigma \approx \frac{2 e \mu_{i 0}\left(\gamma_{c 0}+\gamma_{r 0}\right)}{n_{a 0} \beta} \exp \left(\frac{z}{l_{a}}\right)
$$

With the above parameters one can obtain that for altitudes greater than several tens or hundreds of meters, the aerosol concentration $n_{a}$ decreases so much that the recombination term becomes greater than $\beta n_{i} n_{a}$. In such a case Equation (4) is simplified to

$$
\sigma \approx \frac{2 e \mu_{i 0} \gamma_{c 0}^{1 / 2}}{\alpha^{1 / 2}} \exp \left\{z\left(\frac{1}{H}+\frac{1}{2 l_{c}}\right)\right\}
$$

In this altitude range Equation (6) must coincide with the familiar empirical dependence $\sigma=\sigma_{0} \exp \left(z / z_{0}\right)$ which is valid if $z<3.6 \mathrm{~km}$ [Chalmers 1967]. Whence it follows the approximate relation between the parame- ters: $H^{-1}+\left(2 l_{c}\right)^{-1}=z_{0}^{-1}$. Taking into account that $z_{0}=0.82$ $\mathrm{km}$ we can estimate the unknown parameter $l_{c}$ as follows: $l_{c}=0.5 \mathrm{~Hz}_{0} /\left(H-z_{0}\right) \approx 0.46 \mathrm{~km}$.

The variations of radon content may greatly affect the air conductivity and this effect depends on the aerosol concentration. To illustrate this tendency, the altitude dependences of $\sigma$ calculated from Equation (4) are shown in Figure 1 with lines 1 and 2 for the clean air $\left(n_{a 0}=2 \times 10^{2} \mathrm{~cm}^{-3}\right)$ and with lines 3 and 4 for the dusty air $\left(n_{a 0}=1.5 \times 10^{3} \mathrm{~cm}^{-3}\right)$. In making the plot of $\sigma$ we have used the above parameters and $l_{a}=50 \mathrm{~m}$. As is seen from Figure 1, the air conductivity decreases with an increase in the aerosol concentration, which is in turn resulted from the enhancement of the dustiness of the atmosphere. The implication here is that the majority of the newly formed small ions connect with the aerosol particles which do not contribute to the air conductivity because of their low mobility.

In Figure 1, we have plotted functions $\sigma(z)$ for two different rates of the radon-induced ion production in order to illustrate the radon effect on the air conductivity. The lines 1 and 3 correspond to $\gamma_{r 0}=6 \mathrm{~cm}^{-3} \mathrm{~s}^{-1}$ while the lines 2 and 4 correspond to doubled value of $\gamma_{r 0}$. For example, the similar changes in radon concentration has been observed by Yasuoka et al. [2009] prior to the 1995 Kobe earthquake. Notice that $\gamma_{r}$ varies directly with radon activity concentration which in turn is proportional to radon concentration. It is obvious from Figure 1 that the increase of $\gamma_{r 0}$ causes the increase of the air conductivity by $10-20 \%$. The radon effect disappears (that is, of the order of $l_{r}$ ) at the altitudes above $1 \mathrm{~km}$ in such a way that all the dependences tend to the unified

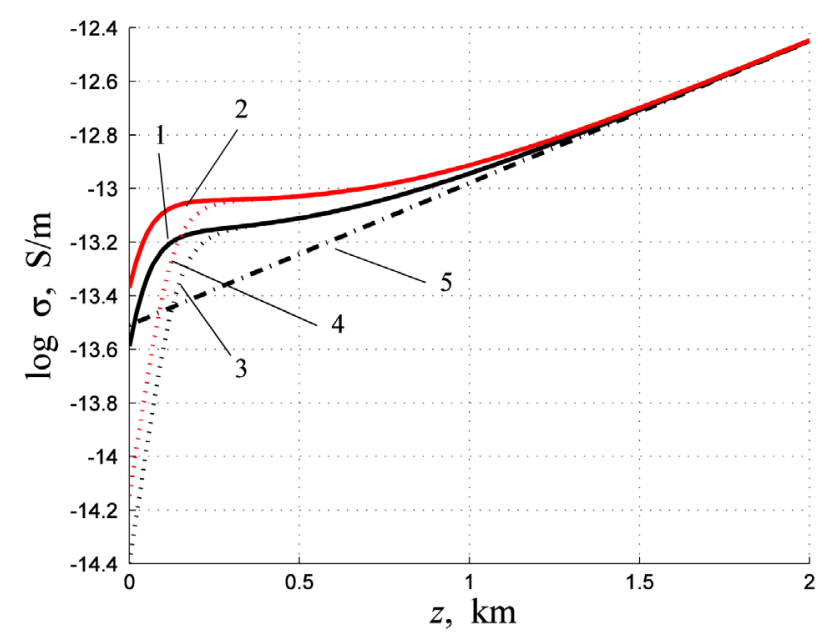

Figure 1. Model distribution of the air conductivity with altitude. The air conductivity for the aerosol number densities $n_{a 0}=2 \times 10^{2}$ $\mathrm{cm}^{-3}$ is shown with lines 1 and 2 while the same value for $n_{a 0}=1.5 \times 10^{3}$ $\mathrm{cm}^{-3}$ is shown with lines 3 and 4 . The sea level rate $\gamma_{r 0}$ of ion production for the lines 2 and 4 is twice as great as that for the lines 1 and 3. Calculation from the empirical equation $\sigma=\sigma_{0} \exp \left(z / z_{0}\right)$ is shown with line 5. 
asymptotic dependence given by Equation (6).

The local variations of radon concentration in the surface layer may affect the total resistance of the atmosphere/ columnar resistance because the surface layer has the greatest air resistance. To illustrate this tendency, we calculate the resistance $R$ per unit of area of the atmospheric surface layer with thickness $z_{s}=3 \mathrm{~km}$

$$
R=\int_{0}^{z_{s}} \frac{d z}{\sigma(z)}
$$

Figure 2 shows the dependence of $R$ on ratio $k=\gamma_{r 0} / \gamma_{c 0}$. In making this plot we have used the above parameters and Equation (4) for $\sigma(z)$. It is generally believed that the typical value of $k$ can vary from 2 to 5 [e.g., Tverskoy 1962, Zhang et al. 2011]. Setting the background value of $k=4$ and using the data by Virk and Singh [1994] and by Yasuoka et al. [2009], we suppose that for seismo-active period this value to be twice as large as that for the background value; that is, $k \approx 8$. As is seen from Figure 2, this can result in the decrease of the surface layer resistance by $15 \%$ for the clean air (line 1 ) and by $25 \%$ for the dusty air (line 2 ). As would be expected, considering the important role played by the surface layer in the atmospheric resistance, these estimates hold true for the total columnar resistance of the atmosphere.

\section{Radon gas activity and electrical structure of the lower atmosphere}

Harrison et al. [2010] have suggested that the abnormal decrease in the atmospheric surface layer resistance caused by an enhancement of the radon volumetric activity can result in an increase in the vertical fair-weather conduction current $j_{a}$ by several units or tens percent. It follows from Maxwell equation that the density of electric charge $\rho=\varepsilon_{0} j_{a} d \sigma^{-1} / d z$, which builds up as a result of the inhomogeneity of the air conductivity, must be proportional to the vertical current density $j_{a}$. This implies that the relative changes of $\rho$ can vary together with $j_{a}$ within the same range. A number of researchers have speculated that the enhancement of the fair-weather conduction current is followed by an increase in the electric charges of water droplets suspended in the air. This effect is assumed to be favorable for the steam condensation which in turn may explain the observation of the so-called earthquake clouds [Rycroft et al. 2008, Rycroft et al. 2012, Harrison et al. 2013].

Here we deal with other radon effects which may affect the electrical structure of the area adjacent to abnormal region. First, we shall estimate a horizontal component of the fair-weather conduction current in the vicinity of the abnormal region with the enhanced

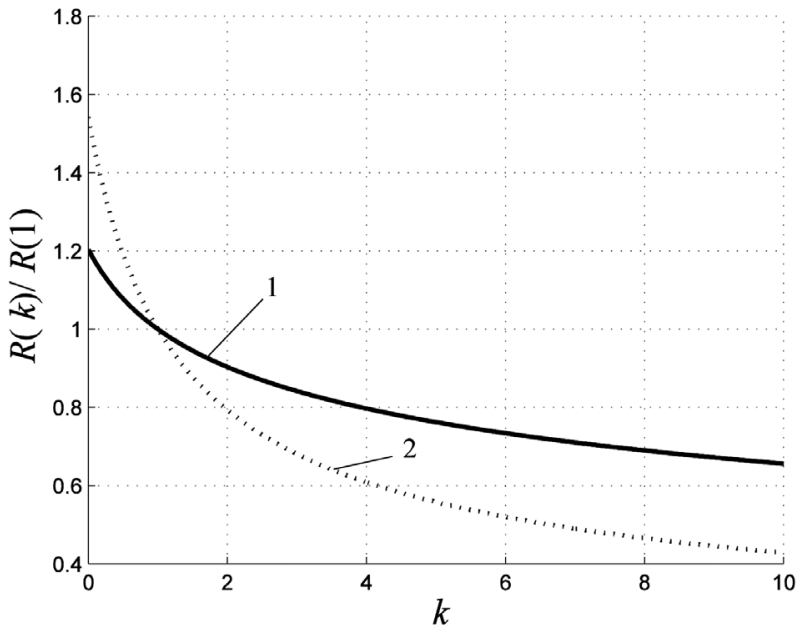

Figure 2. Total columnar resistance $R$ of the atmospheric surface layer versus parameter $k=\gamma_{r 0} / \gamma_{c 0}$. The lines 1 and 2 correspond to aerosol number densities of $2 \times 10^{2} \mathrm{~cm}^{-3}$ and $1.5 \times 10^{3} \mathrm{~cm}^{-3}$, respectively. The plots are normalized to the value of $R$ taken at $k=1$.

radon concentration. Taking into account that the conduction current density is not a function of altitude, the distribution of electric potential with altitude can be written as

$$
\varphi(z)=\varphi_{a} \int_{0}^{z} \frac{d z}{\sigma(z)}\left(\int_{0}^{z_{a}} \frac{d z}{\sigma(z)}\right)^{-1}
$$

where $\sigma(z)$ is given by Equation (4), and $\varphi_{a} \approx 250 \mathrm{kV}$ [e.g., Rycroft et al. 2000] is the potential drop between the bottom of the ionosphere $z=z_{a}$ and the Earth $z=0$. Figure 3 shows the result of numerical integration of Equation (8). As before the lines 1 and 3 correspond to $\gamma_{r 0}=6 \mathrm{~cm}^{-3} \mathrm{~s}^{-1}$ while the lines 2 and 4 correspond to $\gamma_{r 0}=12 \mathrm{~cm}^{-3} \mathrm{~s}^{-1}$. It is obvious from Figure 3 that an increase of the ionization rate $\gamma_{r 0}$ leads to decrease of potential (8) at given altitude. It also can be seen that there are the essential distinctions between background (lines 1 and 3 ) and abnormal (lines 2 and 4) potential distri-

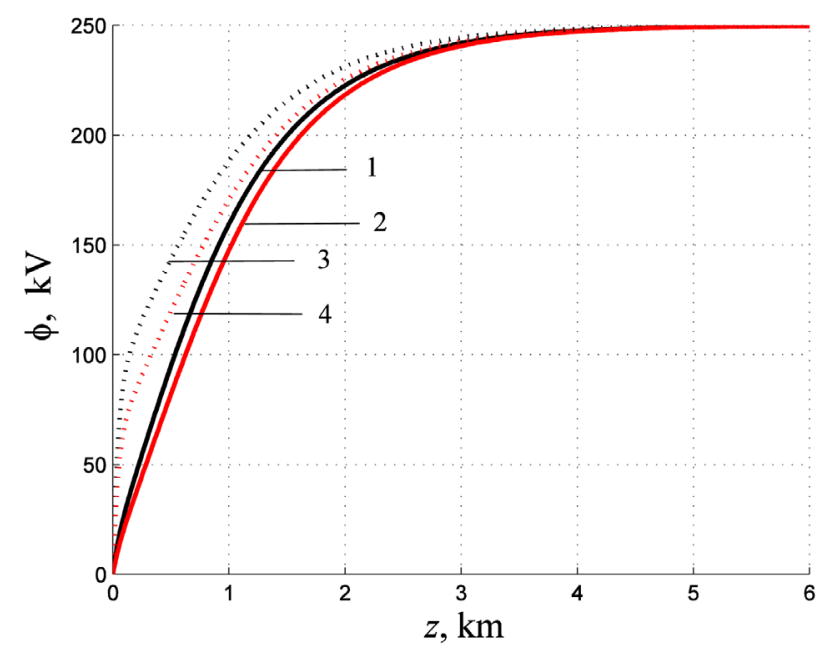

Figure 3. The same as in Figure 1 but for the atmospheric potential. 
butions in the altitude range $02-2 \mathrm{~km}$. In order to highlight this peculiarity, the differences between the corresponding distributions as a function of altitude are shown in Figure 4. Here the line 1 demonstrates the difference between the first and second distributions shown in Figure 3 whereas the line 2 demonstrates the same but for the third and fourth distributions.

This constitutes a mapping of the surface radon activity into space variations of the atmospheric potential at the altitudes of several kilometers. The recent measurements [Kabrt et al. 2014] of radon gas activity concentration distributed over a large area can serve as a basis for the searching of the seismic-induced radon anomaly region. As would be expected, the horizontal electric fields and currents occur near the boundary of the abnormal region where the radon concentration changes from the enhanced value to the background one. The reason is that the height-dependence of potential in the abnormal area is different from that in the surrounding regions. The horizontal field components can be estimated roughly as follows $E_{\|} \sim \delta \varphi / \delta l$ and $j_{\|} \sim \sigma E_{\|}$where $\delta l$ is the horizontal space scale at which the radon number density decreases down to the background level while $\delta \varphi$ is the corresponding potential difference taken at the fixed height. It follows from Figures 1 and 4 that maximum value $\delta \varphi=13-25 \mathrm{kV}$ is achieved at such altitudes where the air conductivity $\sigma=8 \times 10^{-14}-10^{-13} \mathrm{~S} / \mathrm{m}$. Taking these values and choosing $\delta l=10-50 \mathrm{~km}$, we come to the following estimates: $E_{\|} \approx 0.26-2.5 \mathrm{~V} / \mathrm{m}$ and $j_{\|} \approx 0.02-0.25 \mathrm{pA} / \mathrm{m}^{2}$. Since the radon anomalies are subjected to temporal variations around seismic event, the above values can only serve as order-of-magnitude estimates of the possible atmospheric perturbations resulted from the enhanced radon emanation.

A more significant effect can be expected in the vicinity of mesoscale convective systems (MSC) since there is the background atmospheric current well above the fair-weather one. The lateral size and total current of the MSC electrical active region are greater than $150 \mathrm{~km}$ and several tens Ampere, respectively, that is much larger than typical thunderstorm parameters [Davydenko et al. 2004]. A portion of this current can close through the abnormal region of the lower atmosphere with the reduced resistance thereby producing the charge relaxation and changes in electrical structure of MSC.

It is pertinent to note that there are a few possible mechanisms which can provide a coupling between ultra low frequency (ULF) electric field and charge density pulsations in the surface atmospheric layer and the variations of radon activity concentration. The fairweather electric field pulsations have a power-law spec-

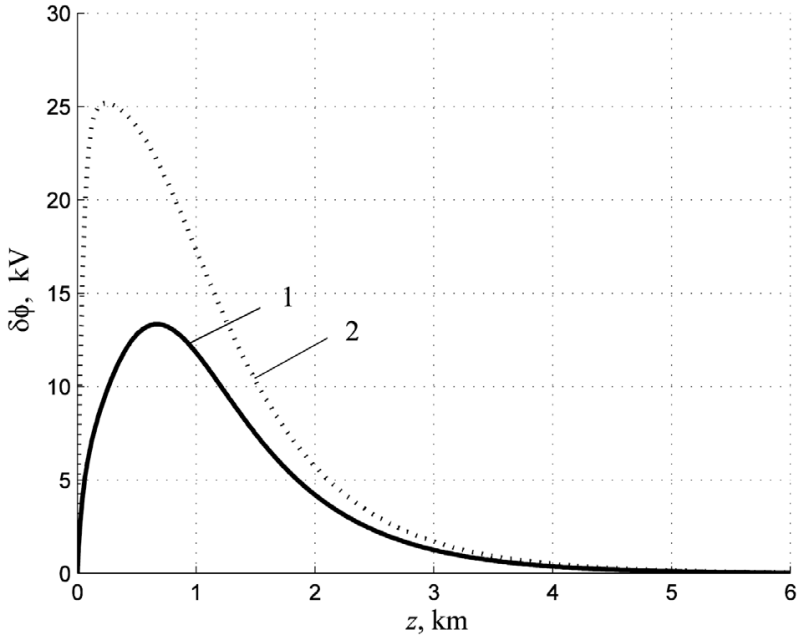

Figure 4. Model calculation of the atmospheric potential difference which might arise in horizontal direction. The potentials were calculated at the same altitudes but for different ion production rates, $\gamma_{r 0}$, which differ by the factor 2 . The lines 1 and 2 correspond to $n_{a 0}=2 \times 10^{2} \mathrm{~cm}^{-3}$ and $n_{a 0}=1.5 \times 10^{3} \mathrm{~cm}^{-3}$, respectively.

trum which falls off with frequency as $f^{-m}$. In the frequency range of $0.004-0.06 \mathrm{~Hz}$ the spectral index $m$ varies from 2 to 2.4 [Yerg and Johnson 1974] while in the range of $0.01-1 \mathrm{~Hz}$ the electrical pulsations and their power spectra can be split into two groups; that is, the so-called "structured spectra" with $m=2.03-3.36$ and "unstructured spectra" with $m=1.23-2.89$ [Anisimov et al. 2002, 2005]. The "structured spectra" has been assumed to be due to aero-electric structures (AESs) involved in the neutral-gas turbulence in the surface layer. The enhancement of radon activity concentration, followed by the production of additional small ions and charged aerosols, can result in the increase of both the space charge and the variance of the charge-density fluctuations $\sigma_{p}^{2}$. According to the model by [Anisimov et al. 2002] the ULF spectrum of electric pulsations is proportional to $\sigma_{p}^{2}$ and thus the variation of radon concentration can affect the spectrum amplitude. We cannot come close to exploring these topics in any detail although one may assume that the spectral index is a function of radon concentration as well.

The release of radon from the soil to the air depends on local rock morphology and genesis including faults, zones of rock fracture and etc. Overall, the radon gas is nonuniformly distributed near the ground surface. This implies that the pre-seismic radon variation in seismo-active region is capable of producing the random spatiotemporal variations of the charge density and electric field in the air. These electric fluctuations appear to have a power-law spectrum in analogous to spectrum of natural ULF magnetic noises produced by random fluctuations of the ionospheric and atmospheric currents [Surkov and Hayakawa 2007, 2008, 2014]. Despite this effect seems to be weak, it can influence 
on the fair-weather spectral index $m$ of natural electric pulsations. So, the changes in the ULF electric spectra amplitude and spectral index can be indicative of preseismic tectonic activity.

\section{Release of radon from the ground and temperature of the atmospheric surface layer}

Statistical analysis of satellite thermal imaging data measured over the high seismicity regions and fault systems in the Earth's crust have shown that the upward infrared (IR) radiation from the atmosphere can exceed the background value as compared to the surrounding regions [Gorny et al. 1993, Tramutoli et al. 2001, Tronin et al. 2002, Ouzounov et al. 2006, Genzano et al. 2009]. This effect is generally interpreted as a by-product of mean temperature increase of the atmospheric surface layer in seismo active regions. The mechanism of the phenomenon was presumed to be due to the release of optically active gases from soil to the air followed by local greenhouse effect [Tronin et al. 2002]. One more a plausible explanation of this phenomenon is the gradual squeezing-out of the groundwater from higher depth towards the ground surface that results from the variations of the tectonic stress in the fault zone. Taking into account that, on the average, the ground temperature increases with depth by $2 \mathrm{~K}$ per $100 \mathrm{~m}$, Surkov et al. [2006] have shown that this leads to the convective heating of the ground surface by a few Kelvin since the temperature of the deep groundwater is higher than the mean temperature of the ground surface.

It was also hypothesized by Pulinets and Ouzounov [2011] and by Pulinets [2011] that the enhancement of the outgoing IR radiation several days before seismic event can be due to the increase in radon concentration in the surface layer. This hypothesis is based on assumption that the radon-producing air ionization gives rise to the formation of so-called large cluster ions with typical sizes $1-3 \mu \mathrm{m}$; that is, as large as the aerosol particles. These large ions can serve as condensation centers triggering the water vapor condensation which in turn is accompanied with the release of the heat of vaporization.

To estimate the maximal value of this effect, we assume that the alpha decay of radon nuclei occurs in a humid air near the ground surface. Let $A$ be the volumetric radon activity and $N_{i}$ be the number of ordinary/primary ion pairs resulted from the scattering of single alpha-particle in the air. In a short time the cluster ions are formed due to attachment of neutral molecules to the primary ions. The polar molecules of air, including the molecules of $\mathrm{H}_{2} \mathrm{O}$, are largely involved in this process. Assuming for the moment that every primary ion gives rise to the formation of cluster ion, the rate of the cluster ion production per unit volume is $A N_{i}$. The volumetric rate of $\mathrm{H}_{2} \mathrm{O}$ molecule attachment to the cluster ions is $A N_{i} N_{w}$, where $N_{w}$ stands for the mean number of $\mathrm{H}_{2} \mathrm{O}$ molecules attached to the single cluster ion. The thermal energy released to the atmosphere per unit time and per unit volume is $A N_{i} N_{w} E_{1}$, where $E_{1}$ is the energy release due to attachment of single $\mathrm{H}_{2} \mathrm{O}$ molecule to the cluster ion. As a rough estimate of $E_{1}$, one may use the value $\lambda / N_{a}$, where $\lambda \approx 41$ $\mathrm{kJ} / \mathrm{mol}$ [Gray 1972] is the molar heat of vaporization for water and $N_{a}$ is the Avogadro number. Let $h_{r}$ be the thickness of the near-surface layer which contains the basic part of atmospheric radon. If the heat capacities of the ground and air are both ignored, the maximal value of upward heat flux density can be estimated as follows: $q_{\max }=A N_{i} N_{w} h_{r} \lambda / N_{a}$.

According to the hypothesis by Pulinets and Ouzounov [2011], the so-called big cluster ions (BCI), "which are actually the cluster ions formed after ionization and ion's hydration", play a crucial role in a water vapor condensation followed by the pre-seismic thermal anomaly. Their numerical estimate is based on assumption that, first, the number of $\mathrm{H}_{2} \mathrm{O}$ molecules attached to the BCI ranges as high as $N_{w}=1.3 \times 10^{11}$; second, the pre-seismic radon activity reaches the value $A=2 \times 10^{3} \mathrm{~Bq} / \mathrm{m}^{3}$ and $N_{i}=4.5 \times 10^{5}$ ion pars [Pulinets and Ouzounov 2011]. Furthermore, it was implicitly supposed that every primary ion is converted to the big ion. Taking $h_{r}=1 \mathrm{~m}$ and substituting the above numerical values of the parameters into the equation for $q_{\max }$, gives the estimate: $q_{\max }=16 \mathrm{~W} / \mathrm{m}^{2}$ which coincides with the numerical estimate given by Pulinets and Ouzounov [2011].

Despite this value is believed to be compatible with the observations of abnormal pre-seismic increase of the upward thermal flux [Ouzounov et al. 2006], such an approach has the serious shortcoming that the rate of the BCI production $A N_{i}$ is dramatically overestimated. Indeed, the radon activity may affect the BCI rate production through the interaction between the radon-produced small ions and the BCI. Taking into account that, first, about 5-20 neutral molecules are attached to the small ion; second, only a part of them are the $\mathrm{H}_{2} \mathrm{O}$ molecules, it becomes apparent that at least about $10^{10}$ small ions needs to create the $\mathrm{BCI}$ about which $N_{w}=1.3 \times 10^{11} \mathrm{H}_{2} \mathrm{O}$ molecules are held. This means that the production rate of the $\mathrm{BCI}$ is smaller than that of the small ions by 10 orders of magnitude.

In other words, the value $A N_{i}=9 \times 10^{8} \mathrm{~m}^{-3} \mathrm{~s}^{-1}$ of the cluster ion production can be applied to the small ions but not to the BCI. Moreover, it seems likely that the parameter $A=2 \times 10^{3} \mathrm{~Bq} / \mathrm{m}^{3}$ used in the cited papers was also overestimated because this value of radon ac- 
tivity concentration is typical for the soil rather than for the atmosphere. It is common knowledge that the world average value of volumetric radon activity, on being measured in the air $1 \mathrm{~m}$ above the ground, varies from 7 to $12 \mathrm{~Bq} / \mathrm{m}^{3}$ though this value can reach $50 \mathrm{~Bq} / \mathrm{m}^{3}$ in areas with radon-laden soil [e.g., Protection against Radon-222 at Home and at Work, 1994]. In addition, we recall that the pre-seismic increase in atmospheric radon activity concentration before Kobe earthquake was as high as $20 \mathrm{~Bq} / \mathrm{m}^{3}$ [Yasuoka et al. 2009]. If this latter value of $A$ is taken, the above estimate of $q_{\max }$ is further lowered by two orders of magnitude.

\section{Discussion and conclusion}

Our theoretical analysis favours the view [e.g., Harrison et al. 2010, 2013, 2014] that the variations of atmospheric radon concentration may affect the columnar resistance of the atmosphere. For example, according to our estimate the pre-seismic increase in the radon activity concentration by two times can result in the local lowering of the standard atmosphere resistance by $10-20 \%$ and approximately the same relative increase in the fair-weather/background conduction current. It follows from our model that the greatest changes of the air conductivity can be expected at the altitudes $100-200 \mathrm{~m}$ in the case of dusty air of high aerosol content. Although, the radon and aerosols appear to have no effect on the air conductivity above 1 $\mathrm{km}$ since the cosmic rays and other mechanisms for air ionization play a major role in this altitudes range.

The enhanced radon release from the ground followed by the variations of the air conductivity may affect the electrical structure of the atmosphere in seismo active regions. For example, not only vertical but also the horizontal components of both the background current density and of the gradient of atmospheric electrical potential can arise near the boundaries of seismo active regions and the Earth crust faults. Our estimates have shown that at the fair weather conditions they can reach the values $0.25 \mathrm{pA} / \mathrm{m}^{2}$ and $2.5 \mathrm{~V} / \mathrm{m}$, respectively. It appears that the horizontal field variations can be even greater than the above values by several orders of magnitude in the presence of strong background current that are typical of mesoscale convective systems and the atmospheric precipitation [Davydenko et al. 2004].

It can be also expected that the increased air ionization due to radon decay has an impact on the aeroelectrical structures thereby producing the pre-seismic changes in spectral indices and amplitudes of ULF electric pulsations resulted from motion of these structures. A closer look at the electric pulsation spectra before seismic events would help us to understand whether this pre-seismic effect takes place or not. If this is the case, the radon-produced air ionization can serve as a plausible candidate for explanation of this effect.

We have analyzed the hypothesis by Pulinets and Ouzounov [2011] that the enhancement of radon release from the ground deeply influences a near-surface process of evaporation and related phenomena such as abnormal variations of upward thermal IR radiation occasionally observed from satellite before earthquake occurrence. The big cluster ions (BCIs) are of great concern in this hypothesis. It should be realized that the properties of the BCI are unusual since they are out of standard classification of ions [e.g., Hirsikko et al. 2011] because of their big size (about $1 \mu \mathrm{m}$ ) and a great number of the attached water molecules (about $10^{11}$ ). Taking into account that the diameter of water molecule is $0.29 \mathrm{~nm}$, it is easy to check that a total volume of these attached molecules are greater than a volume of the individual BCI. In this notation the BCI should be assigned to water droplets rather than to cluster ions. The conditions for the generation of BCI are unknown and, perhaps, the BCI seldom if ever occurs in the actual atmosphere.

However the major problem of this hypothesis is that the production rate of the $\mathrm{BCI}$ needs to be as high as and even greater than that of small ions in order to explain the observed data. According to our estimates, the air molecule ionization by atmospheric radon decay must lead to the generation of approximately $10^{9} \mathrm{BCI}$ per unit volume and per unit time in order to produce the upward thermal flux about $16 \mathrm{~W} / \mathrm{m}^{2}$. This implies that every primary ion originated from alpha particle scattering would end up as the BCI. Besides the atmospheric radon activity concentration used by Pulinets and Ouzounov [2011] is two orders of magnitude greater than the actual one. Taken together, it makes this hypothesis very questionable.

We can conclude that, in light of our study, the change in the air resistance of surface atmospheric layer by several tens percent is currently the only plausible radon effect which can be predicted with confidence. Although one might expect certain changes in the electric structure of the lower atmosphere. A network of ground-based electric sensors covered seismo-active region is necessary in order to monitor the electrical field perturbations due radon anomaly. Despite these effects seem to be sensitive to meteorological conditions, the electrical perturbations can be detectable at the ground level if it is granted that daily and seasonal variations of fair-weather atmospheric currents and electric fields are eliminated. It appears that further experiments are necessary to provide us with evidence both in support of and against a concept that other pre-seismic radon effect actually exists. 
Acknowledgements. This study was supported by the grant no. 13-05-12091 from RFBR.

\section{References}

Anisimov, S.V., E.A. Mareev, N.M. Shikhova and E.M. Dmitriev (2002). Universal spectra of electric field pulsations in the atmosphere, Geophys. Res. Lett., 29 (24), 2217-2220; doi:10.1029/2002GL015765.

Anisimov, S.V., E.A. Mareev, N.M. Shikhova, A.E. Sorokin and E.M. Dmitriev (2005). On the electrodynamical characteristics of the fog, Atmosph. Res., 76 (1-4), 16-28.

Chalmers, J.A. (1967). Atmospheric electricity, 2nd ed., Pergamon press, New York.

Cicerone, R.D., J.E. Ebel and J. Britton (2009). A systematic compilation of earthquake precursors, Tectonophysics, 476, 371-396.

Davydenko, S.S., E.A. Mareev, T.C. Marshall and M. Stolzenburg (2004). Calculation of electric fields and currents of mesoscale convective systems and their influence on the global electric circuit, J. Geophys. Res., D109, 11103-11110; doi:10.1029/2003JD003832.

Geller, R.J. (1997). Earthquake prediction: a critical review, Geophys. J. Int., 131, 425-450.

Genzano, N., C. Aliano, R. Corrado, C. Filizzola, M. Lisi, G. Mazzeo, R. Paciello, N. Pergola and V. Tramutoli (2009). RST analysis of MSG-SEVIRI TIR radiances at the time of the Abruzzo 6 April 2009 earthquake, Nat. Hazards Earth Syst. Sci., 9, 20732084.

Gorny, V.I., B.B. Shilin and G.I. Yasinsky (1993). Thermal Aerospace Survey, Nedra, Moscow (in Russian).

Gray, D.E. (1972). American Institute of Physics Handbook, 3rd ed., McGraw-Hill Book Company, 2364 p.

Harrison, R.G., K.L. Aplin and M.J. Rycroft (2010). Atmospheric electricity coupling between earthquake regions and the ionosphere, J. Atmos. Sol.-Terr. Phys., 72, 376-381.

Harrison, R.G., K.L. Aplin and M.J. Rycroft (2013). Earthquake-cloud coupling through the global atmospheric electric circuit, Nat. Hazards Earth Syst. Sci. Discuss., 1, 7271-7283; doi:10.5194/nhessd-17271-2013.

Harrison, R.G., K.L. Aplin and M.J. Rycroft (2014). Brief Communication: Earthquake-cloud coupling through the global atmospheric electric circuit, Nat. Hazards Earth Syst. Sci., 14, 773-777; doi:10.5194/nhess-14773-2014.

Hirsikko, A., T. Nieminen, S. Gagné, K. Lehtipalo, H.E. Manninen, M. Ehn, U. Hõrrak, V.-M. Kerminen, L. Laakso, P.H. McMurry, A. Mirme, S. Mirme, T. Petäjä, H. Tammet, V. Vakkari, M. Vana and M. Kulmala (2011). Atmospheric ions and nucleation: a re- view of observations, Atmos. Chem. Phys., 11, 767798; doi:10.5194/acp-11-767-2011.

Inan, S., T. Akgül, C. Seyis, R. Saatçılar, S. Baykut, S. Ergintav and M. Bas (2008). Geochemical monitoring in the Marmara region (NW Turkey): a search for precursors of seismic activity, J. Geophys. Res., 113, B03401; doi:10.1029/2007JB005206.

Israël, H. (1970). Atmospheric Electricity, Israel Program for Scientific Translations, Jerusalem, 1, 317 p.

Ivlev, L.S., Yu.A. Dovgalyuk (1999). Physics of atmospheric aerosol systems, St Petersburg, St Petersburg State University, 194 p. (in Russian).

Kabrt, F., C. Seidel, A. Baumgartner, H. Friedmann, F. Rechberger, M. Schuff and F.J. Maringer (2014). Radon soil gas measurements in a geological versatile region as basis to improve the prediction of areas with a high radon potential, Rad. Prot Dos., 160 (1-3), 217-221; doi:10.1093/rpd/ncu086.

Kondratyev, K.Ya., L.S. Ivlev, V.F. Krapivin and C.A. Varotsos (2006). Atmospheric Aerosol Properties: Formation, Processes and Impacts, Springer/PRAXIS, Chichester, UK, 572 p.

Ouzounov, D., M. Bryant, T. Logan, S. Pulinets and P. Taylor (2006). Satellite thermal IR phenomena associated with some of the major earthquakes in 1999-2003, Phys. Chem. Earth, 31, 154-163.

Pierce, E. (1976). Atmospheric electricity and earthquake prediction, Geophys. Res. Lett., 3 (3), 185-188.

Protection against Radon-222 at Home and at Work (1994). Annals of the ICRP, No. 65, Oxford, Pergamon.

Pulinets, S. (2011). The synergy of earthquake precursors, Earthq. Sci., 24, 535-548.

Pulinets, S., and D. Ouzounov (2011). Lithosphere-Atmosphere-Ionosphere Coupling (LAIC) model - An unified concept for earthquake precursors validation, J. Asian Earth Sci., 41, 371-382.

Rycroft, M.J., S. Israelsson and C. Price (2000), The global atmospheric electric circuit, solar activity and climate change, J. Atmos. Sol.-Terr. Phys., 62, 15631576.

Rycroft, M.J., R.G. Harrison, K.A. Nicoll and E.A. Mareev (2008). An overview of Earth's global electric circuit and atmospheric conductivity, Space Sci. Rev., 137 (1-4), 83-105; ISSN 0038-6308, doi: 10.1007/s112 14-008-9368-6.

Rycroft, M.J., K.A. Nicoll, K.L. Aplin and R.G. Harrison (2012). Recent advances in global electric circuit coupling between the space environment and the troposphere, J. Atmos. Sol.-Terr. Phys., 90-91, 198-211.

Surkov, V.V., O.A. Pokhotelov, M. Parrot and M. Hayakawa (2006). On the origin of stable IR anomalies detected by satellite above seismo-active regions, 
Phys. Chem. Earth, 31, 164-171.

Surkov, V.V., and M. Hayakawa (2007). ULF electromagnetic noise due to random variations of background atmospheric current and conductivity, J. Geophys. Res., 112, D11116; doi:10.1029/2006JD007788.

Surkov, V.V., and M. Hayakawa (2008). Natural electromagnetic ULF noise due to fluctuations of ionospheric currents, J. Geophys. Res., 113, A11310; doi:10. 1029/2008JA013196.

Surkov, V., and M. Hayakawa (2014). Ultra and Extremely Low Frequency Electromagnetic Fields, Springer Geophysics Series, XVI, Springer, 486 p.

Toutain, J.-P., and J.-C. Baubron (1998). Gas geochemistry and seismotectonics: a review, Tectonophysics, 304, 1-27.

Tramutoli, V., D. Bello, G.N. Pergola and S. Piscitelli (2001). Robust satellite technique for remote sensing of seismically active areas, Annali di Geofisica, 44, 295-312.

Tronin, A.A., M. Hayakawa and O.A. Molchanov (2002). Thermal IR satellite application for earthquake research in Japan and China, J. Geodynamics, 33, 519-534.

Tverskoy, P.N. (1962). Meteorology (Physics of the atmosphere), Gydrometeoizdat, Leningrad (in Russian).

Virk, H.S., and B. Singh (1994). Radon recording of Uttarkashi earthquake, Geophys. Res. Lett., 21, 737742.

Wåhlin, L. (1994). Elements of fair weather electricity, J. Geophys. Res., 99, D10767-D10772.

Yasuoka, Y., Y. Kawada, H. Nagahama, Y. Omori, T. Ishikawa, S. Tokonami and M. Shinogi (2009). Preseismic changes in atmospheric radon concentration and crustal strain, Phys. Chem. Earth, 34, 431-434.

Yerg, D.G., and K.R. Johnson (1974). Short-period fluctuations in the fairweather electric field, J. Geophys. Res., 79, 2177-2184.

Zhang, K., J. Feichter, J. Kazil, H. Wan, W. Zhuo, A. D. Griffiths, H. Sartorius, W. Zahorowski, M. Ramonet, M. Schmidt, C. Yver, R. E. M. Neubert and E.-G. Brunke (2011). Radon activity in the lower troposphere and its impact on ionization rate: a global estimate using different radon emissions, Atmos. Chem. Phys., 11, 7817-7838; doi:10.5194/acp-117817-2011.

Corresponding author: Vadim Surkov,

National Research Nuclear University MEPhI, Moscow, Russia; email: surkovvadim@yandex.ru. 